\title{
Signal waveform analysis for all-optical waveform converter by adjusting the power and the wavelength of assist light
}

\author{
Motoharu Matsuura $^{1 a)}$, Yuki Kishikawa ${ }^{2}$ Naoto Kishi $^{1}$, \\ and Tetsuya Miki ${ }^{1}$ \\ ${ }^{1}$ Department of Information and Communication Engineering, \\ University of Electro-Communications, \\ 1-5-1 Chofugaoka, Chofu, Tokyo 182-8585, Japan \\ ${ }^{2}$ NTT DoCoMo, Inc., \\ 2-11-1 Nagata-cho, Chiyoda-ku, Tokyo 100-6150, Japan \\ a) matsuura@ice.uec.ac.jp
}

\begin{abstract}
We analyze signal waveform generated by a semiconductor optical amplifier (SOA)-based switch with a delayed-interferometric (DI) configuration by controlling an assist light under gain or transparent wavelength. To take advantage of the rectangular-shape waveform generated by the switch, we investigate and optimize the waveform quality by means of both $Q$-factor and concordance rate to rectangularshape of the converted waveform.
\end{abstract}

Keywords: all-optical wavelength conversion, waveform conversion, semiconductor optical amplifiers (SOAs), patterning effect, assist light

Classification: Photonics devices, circuits, and systems

\section{References}

[1] K. E. Stubkjaer, "Semiconductor optical amplifier-based all-optical gates for high-speed optical processing," IEEE J. Select. Topics Quantum Electron., vol. 6, no. 6, pp. 1428-1435, Nov./Dec. 2000.

[2] J. P. Sokoloff, P. R. Prucnal, I. Glesk, and M. Kane, "A terahertz optical asymmetric demultiplexer (TOAD)," IEEE Photon. Technol. Lett., vol. 5, no. 7, pp. 787-790, July 1993.

[3] R. J. Manning and D. A. O. Davies, "Three-wavelength device for alloptical signal processing," Opt. Lett., vol. 19, no. 12, pp. 889-891, June 1994.

[4] M. Usami, M. Tsurusawa, and Y. Matsushima, "Mechanism for reducing recovery time of optical nonlinearity in semiconductor laser amplifier," Appl. Phys. Lett., vol. 71, no. 22, pp. 2657-2659, May 1998.

[5] B. Mikkelsen, K. S. Jepsen, M. Vaa, H. N. Poulsen, K. E. Stubkjaer, R. Hess, M. Duelk, W. Vogt, E. Gamper, E. Gini, P. A. Besse, H. Melchior, S. Bouchoule, and F. Devaux, "All-optical wavelength converter scheme for high speed RZ signal formats," Electron. Lett., vol. 33, no. 25, pp. 2137-2139, Dec. 1997. 
[6] M. Matsuura, N. Kishi, and T. Miki, "Performance improvement of optical RZ-receiver by utilizing an all-optical waveform converter," OSA Opt. Expr., vol. 13, no. 13, pp. 5074-5079, June 2005.

[7] H. Wang, J. Wu, and J. Lin, "Performance analysis on terahertz optical asymmetric demultiplexer with assist light injection," Opt. Commun., vol. 256, pp. 83-97, 2005.

[8] Y. Kishikawa, M. Matsuura, and N. Kishi, "Influence of patterning effect on the all-optical delayed-interferometric switch using semiconductor optical amplifier," in Proc. Int. Symp. on Contemporary Photon. Technol. (CPT 2006), Last-Minute 6, Tokyo, Japan, Jan. 2006.

[9] R. J. Manning, A. D. Ellis, J. Poustie, and K. J. Blow, "Semiconductor laser amplifiers for ultrafast all-optical signal processing," J. Opt. Soc. Am. B, vol. 14, no. 11, pp. 3204-3216, Nov. 1997.

[10] J. Leuthold, M. Mayer, J. Eckner, G. Guekos, and H. Melchior, "Material gain of bulk $1.55 \mu \mathrm{m}$ InGaAsP/InP semiconductor optical amplifiers approximated by a polynomial model," J. Appl. Phys., vol. 87, no. 1, pp. 618-620, Jan. 2000.

[11] J. L. Pleumeekers, M. Kauer, K. Dreyer, C. Burrus, A. G. Dentai, S. Shunk, J. Leuthold, and C. H. Joyner, "Acceleration of gain recovery in semiconductor optical amplifiers by optical injection near transparency wavelength," IEEE Photon. Technol. Lett., vol. 14, no. 1, pp. 12-14, Jan. 2002 .

\section{Introduction}

All-optical signal processing is one of the important technologies for future ultrafast photonic networks. In particular, SOA-based devices have some advantages such as power consumption, low switching energy, and compact size [1]. However, one drawback of such devices is the operation speed restricted by long carrier semiconductor recovery time. In case of wavelength conversions, this causes the degradation of output signal quality due to waveform distortion with long trailing edge and patterning effect. The long trailing edge can be cancelled using a DI configuration [2], whereas the long recovery time must be reduced to suppress the patterning effect. A practical way to reduce the recovery time is to increase the optical power injected into SOA using an assist light with the wavelength of gain [3] or transparent region [4].

Another advantage of DI configuration is output pulse-width controllability of the converted signal by adjusting the time delay between the arms in the interferometer. Moreover, this feature is applicable to an all-optical waveform converter to generate an output waveform with a rectangular-like shape [5]. Previously, we have applied this technique to optical receiver design and successfully improved the receiver performance such as receiver sensitivity and phase margin [6]. In this scheme, the slower recovery is required to generate a better rectangular-waveform, whereas it causes stronger patterning effect. Hence, to minimize both waveform distortion and patterning effect of the converted signal, the recovery time must be optimized to obtain best signal quality for all-optical waveform conversion. Although the waveform 


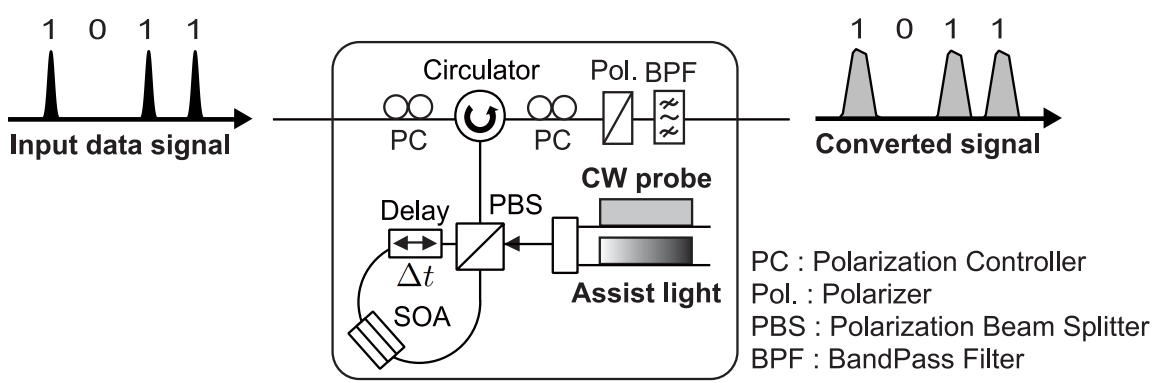

(a)

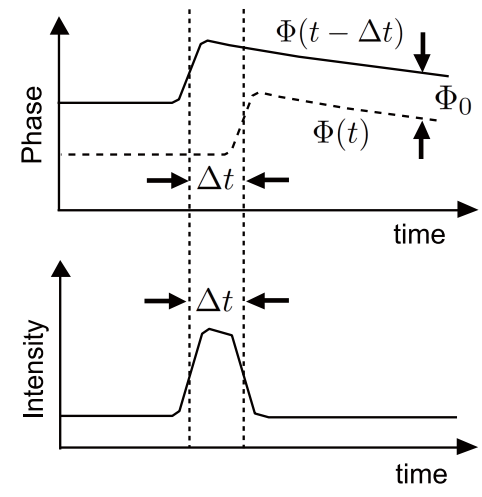

(b)

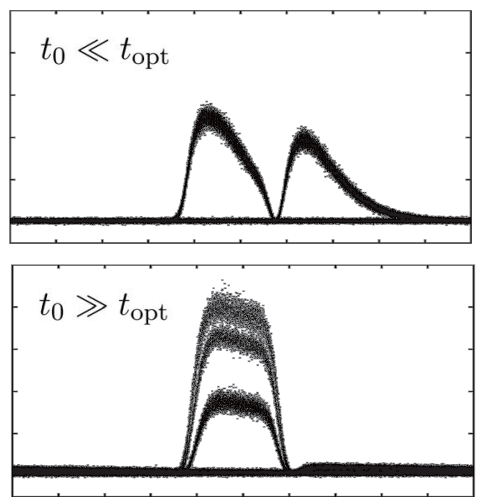

(c)

Fig. 1. (a) Configuration of all-optical waveform converter. (b) Temporal phase and intensity changes of the probe beams. (c) Examples of eye-pattern.

analysis as a function of the recovery time has already been reported [7], no optimized waveform analysis is reported taking into account the trade-off between rectangular-waveform and patterning effect. In this paper, we analyze the signal quality to optimize the waveform of the converted signal based on both $Q$-factor and concordance rate to rectangular-shape [8].

\section{Trade-off between rectangular-waveform and patterning ef- fect of converted signal}

The configuration of all-optical waveform converter is depicted in Fig. 1 (a). This consists of a terahertz optical asymmetric demultiplexer (TOAD) configuration with a SOA that is asymmetrically placed in the Sagnac loop to neutralize slow recovery time $[2,6]$. In Fig. $1(\mathrm{~b})$, the co- and counterpropagating continuous-wave $(\mathrm{CW})$ probe beams into the loop experience the phase change in the SOA with the time delay $\Delta t$, which is determined by the delay line. After traveling into the loop, the long trailing edges are neutralized, and the combined phase change is converted into the intensity modulation by means of the interferometric configuration in this converter. Therefore, an arbitrary output pulse-width is obtained by adjusting the time delay $\Delta t$. On the other hand, the recovery time $t_{0}$ of the employed SOA also gives a strong influence to the output waveform.

Figure $1(\mathrm{c})$ shows the examples of the output eye-pattern for different recovery times. When the recovery time $t_{0}$ is much shorter than the opti- 
mum recovery time $t_{\mathrm{opt}}\left(t_{0} \ll t_{\mathrm{opt}}\right)$, the non-destructive interferometric pulse component behind the main pulse becomes large. Thus, the converted waveform is drastically distorted as shown in the upper part of Fig. 1(c). This behavior is remarkably observed when the time delay $\Delta t$ is much longer than the pulse-width of the input signal. In contrast, when the recovery time is much longer $\left(t_{0} \gg t_{\mathrm{opt}}\right)$, the signal quality is degraded due to the patterning effect, although the more rectangular-like waveform can be obtained. This means that there exists a trade-off between the waveform distortion and patterning effect of the converted signal. Therefore, it is necessary to optimize the recovery time by adjusting the input power of the assist light in order to obtain the best signal quality of the converted signal.

\section{Numerical model and results}

To calculate the output waveform of the converted signal, we modeled the mechanism of nonlinear switching using a rate equation of the carrier density in SOA [9].

$$
\begin{aligned}
\frac{d N}{d t}=\frac{J}{e A l}-\frac{N}{t_{0}} & -\frac{P_{\mathrm{d}}(t) \cdot \Gamma \cdot G\left(N, \lambda_{\mathrm{d}}\right)}{h \nu_{\mathrm{d}} A} \cdot\left(N-N_{\mathrm{d}}\right) \\
& -\frac{P_{\mathrm{p}}(t) \cdot \Gamma \cdot G\left(N, \lambda_{\mathrm{p}}\right)}{h \nu_{\mathrm{p}} A} \cdot\left(N-N_{\mathrm{p}}\right) \\
& -\frac{P_{\mathrm{a}}(t) \cdot \Gamma \cdot G\left(N, \lambda_{\mathrm{a}}\right)}{h \nu_{\mathrm{a}} A} \cdot\left(N-N_{\mathrm{a}}\right)
\end{aligned}
$$

where $N$ is the carrier density, $J$ is the injection current, $e$ is the electronic charge, $A$ and $l$ are the cross section area and length of the employed SOA, respectively, $P_{\mathrm{d}, \mathrm{p}, \mathrm{a}}(t)$ are the injected powers of the input data signal, probe signal, and assist light, respectively, $\Gamma$ is the mode confinement factor, $G\left(N, \lambda_{\mathrm{d}, \mathrm{p}, \mathrm{a}}\right)$ are the gain coefficients with wavelength dependence approximated by a polynomial model [10], $\nu_{\mathrm{d}, \mathrm{p}, \mathrm{a}}$ and $N_{\mathrm{d}, \mathrm{p}, \mathrm{a}}$ are the optical frequency and carrier density at transparency of each signal, respectively. The output waveform $P_{\operatorname{sig}}(t)$ of the converted signal is given by

$$
P_{\text {sig }}(t)=\frac{1}{2}\left[1+\cos \left(\Phi(t)-\Phi(t-\Delta t)+\Phi_{0}\right)\right]
$$

where $\Phi(t)$ and $\Phi(t-\Delta t)$ are the temporal phase changes of the co- and counter-propagating probe beams, respectively, $\Phi_{0}$ is the phase offset as shown in Fig. 1(b). These phase changes are obtained from the change in the carrier density described in Eq. (1), while the phase offset is optimized for each condition in order to achieve the best extinction ratio of the converted waveform. It should be noted that the temporal response depending on the injecting direction to the SOA [7] is not considered in this simulation. These parameter values are experimentally determined from the measured gain properties of the employed SOA and its output waveforms. Moreover, to fit the experimental condition, the proper amplified spontaneous emission noise of the SOA and receiver noise are added to the simulated waveform.

To compare the signal quality of the converted waveform, we plotted the eye-pattern of the simulated waveform. In this simulation, the bit-rate and 


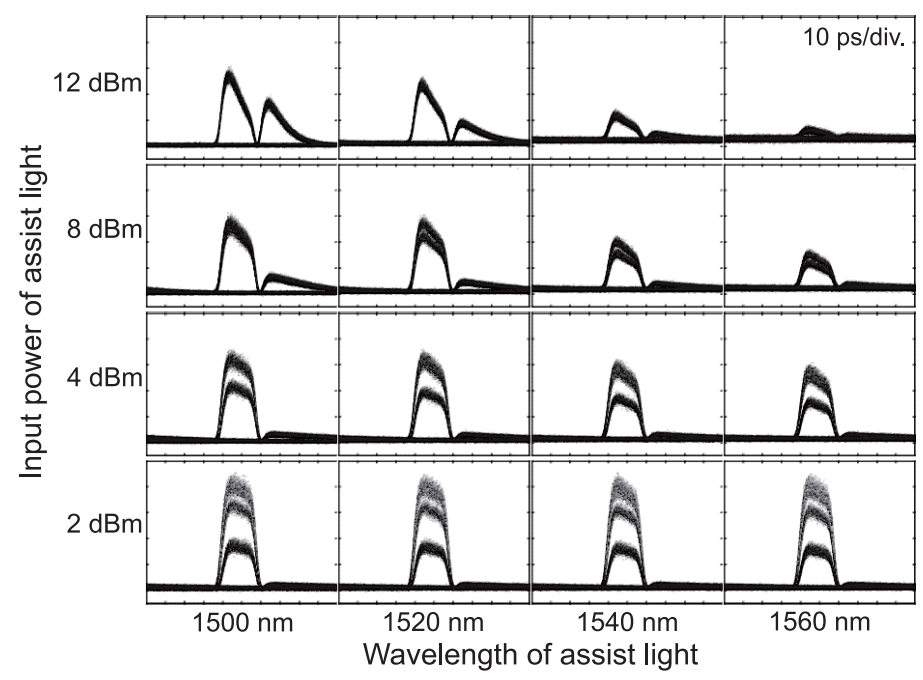

Fig. 2. Simulated output eye-patterns with changing the input power and wavelength of the assist light.

pulse-width of the input data signal are $10-\mathrm{Gb} / \mathrm{s}$ and $5.5 \mathrm{ps}$, respectively. The wavelength and injected average power into the converter of the data signal are set to $1550 \mathrm{~nm}$ and $4.6 \mathrm{dBm}$, respectively, while the parameters of the probe signal are $1560 \mathrm{~nm}$ and $-10 \mathrm{dBm}$, respectively. The data pattern consists of a pseudorandom binary sequence with the bit length of 128 . The time delay $\Delta t$ is set to $20 \mathrm{ps}$. The gain peak and transparent wavelengths of the SOA are $1560 \mathrm{~nm}$ and $1490 \mathrm{~nm}$, respectively.

Figure 2 shows the output eye-patterns of the converted signal with changing the input power and wavelength of the assist light. When the input power of the assist light is small, strong patterning effect is observed regardless the assist wavelength. In contrast, the patterning effect becomes weaker as the input assist power becomes larger. On the other hand, the extinction ratio of the converted waveform is degraded under gain wavelength injection of the assist light. This is due to the gain compression effect among the data signal, probe signal, and assist light. These results show that the near transparent assist light injection enables us to reduce the recovery time without degrading the extinction ratio of the converted waveform [11].

As mentioned in the Section 1, the waveform quality of the converted signal must be estimated by considering rectangular-shape waveform generation. Thus, we investigated the signal quality using both the $Q$-factor and concordance rate $C_{r}$ to rectangular-shape defined as

$$
\begin{gathered}
Q=\frac{\mu_{1}-\mu_{0}}{\tau_{1}+\tau_{0}} \\
C_{\mathrm{r}}=1-\frac{\int_{0}^{T}\left|P_{\mathrm{opt}}(t)-P_{\mathrm{sig}}(t)\right| d t}{\int_{0}^{T} P_{\mathrm{opt}}(t) d t}
\end{gathered}
$$

where $\mu_{1,0}$ and $\tau_{1,0}$ are the decision thresholds and the standard deviations at "1 (mark)" and "0 (space)" levels, respectively, $P_{\text {opt }}(t)$ is the ideal rectangular-shape waveform, $T$ is the bit period of $10-\mathrm{Gb} / \mathrm{s}(T=100 \mathrm{ps})$. 


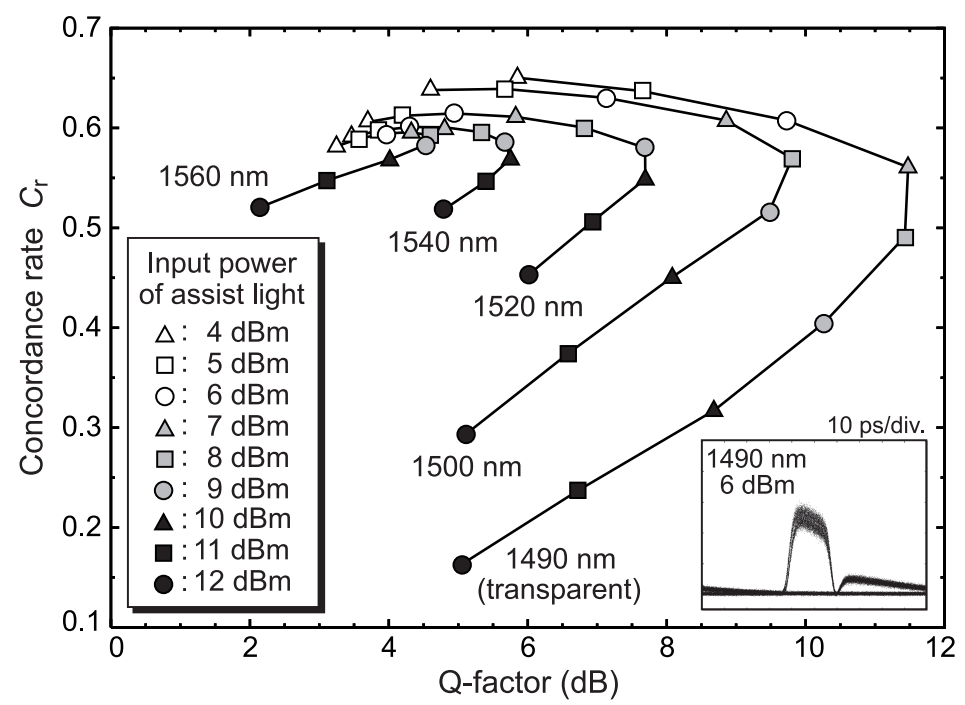

Fig. 3. $Q$-factor versus concordance rate $C_{r}$ for different input powers and wavelengths of assist light.

Figure 3 shows the relation between the calculated $Q$-factor and the concordance rate $C_{r}$ for various assist light conditions. It can be clearly seen that the obtained characteristics at each assist wavelength have the trade-off between the rectangular-waveform and signal quality based on the $Q$-factor of the converted signal as shown in Fig. 2. The optimum assist light power to obtain both high values of the $Q$-factor and $C_{r}$ is around $6 \mathrm{dBm}$ to $8 \mathrm{dBm}$ under the transparent injection of the assist light at the wavelength of $1490 \mathrm{~nm}$. It should be noted that the optimum signal quality should be evaluated primarily by either $C_{r}$ and $Q$-factor depending on the application of the converted signal. For example, $C_{r}$ is more important if a better rectangular-shape waveform is required, whereas $Q$-factor is more important if a higher $Q$-value is required. The inset shows the output eye-pattern of the converted signal for the transparent assist light with $6 \mathrm{dBm}$ input power. This indicates clear eye-opening with a rectangular-waveform.

\section{Conclusion}

The signal quality generated by an all-optical waveform converter was theoretically investigated by controlling the assist light under gain or transparent wavelength. The obtained results are useful for optimizing the signal performance of all-optical waveform conversion. 